\title{
Beta-Thalassemia major and pregnancy
}

\author{
Gulino FA ${ }^{1}$, Vitale $\mathrm{SG}^{2}$, Fauzia $\mathrm{M}^{1}$, Cianci $\mathrm{S}^{2}$, Pafumi $\mathrm{C}^{1}$, Palumbo $\mathrm{MA}^{1}$ \\ Center of Physiopathology of Reproduction, Dpt of Surgery, S. Bambino Hospital, University of Catania, Italy. \\ vitalesalvatore@hotmail.com
}

\begin{abstract}
Objective: It was studied the clinical management and the medical outcomes of 6 pregnancies in 5 women affected by Beta Thalassemia major, based on last guidelines and pharmacological treatments. Background: Paediatric Department and Department of Obstetrics and Gynaecology of the University of Catania. Methods: These patients were taken among a group of 116 women affected by beta-thalassemia major divided into three subgroups, according to the characteristics of their menstrual cycle: 1) women with primitive amenorrhoea, 2) women with secondary amenorrhoea and 3) women with normal menstruation. Only one woman, affected by primitive amenorrhoea, needed the induction of ovulation. An accurate and detailed pre-pregnancy assessment was effected before each conception. This was constituted by a series of essays, including checks for diabetes and hypothyroidism, for B and C hepatitis and for blood group antibodies. Moreover were evaluated: cardiac function, rubella immunity and transaminases. Other pregnancy monitoring, and cares during labour and delivery were effected according to usual obstetrics practice.

Results: All the women were in labour when they were 38 week pregnant, and the outcome were six healthy babies born at term. There were no complications related to the pregnancy and to the immediate outcome after delivery. Conclusions: The improvements of current treatments, especially in the management of iron deposits, the prolongation of survival rate, will result in a continuous increase of pregnancies in thalassemic women. Pregnancy is now a real possibility for women affected by such disease. Although numerous complications can occur, vigilant monitoring by both experienced obstetricians and hematologists can lead to successful pregnancy outcomes (Tab. 1, Fig. 1, Ref. 16). Full Text in PDF www.elis.sk. Key words: beta-thalassemia major, fertility, pregnancy, haemoglobinopathia, amenorrhoea.
\end{abstract}

Beta-thalassemias ( $\beta$-thalassemias) are a group of inherited blood disorders caused by reduced or absent synthesis of the beta chains of haemoglobin resulting in variable phenotypes ranging from severe anemia to clinically asymptomatic individuals.

The phenotypes of homozygous or genetic heterozygous compound beta-thalassemias include thalassemia major and thalassemia intermedia. Individuals with thalassemia major usually come to medical attention within the first two years of life and require regular RBC transfusions to survive.

Clinical presentation of thalassemia major occurs between 6 and 24 months. Affected infants fail to thrive and become progressively pale. Feeding problems, diarrhea, irritability, recurrent bouts of fever, and progressive enlargement of the abdomen caused by spleen and liver enlargement may occur (1).

The ideal management of thalassemia involves a multidisciplinary therapeutic team approach (2) and should be preferably done at a comprehensive thalassemia care center having a team of pediatric hematologist, pediatrician, transfusion medicine special-

${ }^{1}$ Center of Physiopathology of Reproduction, Dpt of Surgery, S. Bambino Hospital, University of Catania, Italy, and ${ }^{2}$ Azienda Ospedaliero-Universitaria "Policlinico-Vittorio Emanuele" G. Rodolico - Operative Unit of Obstetrics and Gynaecology- University of Catania, Italy

Address for correspondence: S.G. Vitale, Dr, Viale della Liberta No. 212, 95129 Catania (CT), Italy.

Phone: +39.3479354575 ist, endocrinologist, psychologist, social worker, and the backup of a well-equipped blood bank (3).

If a regular transfusion program that maintains a minimum $\mathrm{Hb}$ concentration of 9.5 to $10.5 \mathrm{~g} / \mathrm{dL}$ is initiated, growth and development tends to be normal up to 10 to 12 years (2).

Gonadal function is impaired in the majority of the patients affected by the homozygous kind of this disease and, especially in females, this is expressed either by a delay in the onset of puberty and menstruation or by secondary amenorrhoea (4). The commonest abnormality was hypogonadotropic hypogonadism, but the relatively high prevalence of other endocrine impairments, causing multiple endocrinopathies, is also well recognised. These kind of endocrine complications are directly related to the serum ferritin level (5).

For delayed puberty in girls, therapy may start with the administration of ethinyl estradiol (2.5-5 $\mu$ g daily) for 6 months, followed by hormonal reassessment. If spontaneous puberty does not occur within 6 months, ethinyl estradiol should be used at increasing dosages (from 5-10 $\mu \mathrm{g}$ daily) for 12 months. If breakthrough uterine bleeding does not occur, a low oestrogenprogesterone hormone replacement is recommended (6). When there is a lack of pubertal progression over a year or longer (arrested puberty) oestrogenprogesterone replacement therapy in females is indicated.

Nowadays, thanks to improved paediatric and haematological cares, patients affected by Cooley disease may live on to be forty or fifty, and can enjoy a quite regular standard of life. So that, 
maintaining a strict therapeutic regimen, women regularly transfused and well chelated can attempt to become pregnant (7-9).

In this study we describe the evolution and the successful outcome of six at term pregnancies ruled out by five women affected by transfusion dependent beta Thalassemia. In reviewing the cares and the complications during and after their pregnancies, we discuss the issue concerning the appropriate preconception counselling of such patients.

\section{Methods}

For several years we have been following 116 women affected by transfusion dependent beta Thalassemia major, diagnosed when they were between 6 and 8 months old at the Paediatric Department of the University of Catania, from the endocrine and reproductive point of view. All of them were aged between 22 to 32 and received a blood transfusion every 15-20 days, in order to maintain the haemoglobin concentration above $10 \mathrm{~g} / \mathrm{dl}$; and were in strict desferrioxamine chelating therapy, to avoid excessive iron overloads. The patients have been divided into three groups according to their first and successive menstruation characteristics: 1) patients with primitive amenorrhoea, 2) patients with secondary amenorrhoea and 3) patients with normal menstruation. In detail 23 of these women, got married: 5 were affected by primitive amenorrhoea; 6 were affected by secondary amenorrhoea and 12 women had normal menstruation. Finally six pregnancies occurred among these 23 women: two among the patients affected respectively by primitive and secondary amenorrhoea, and four were carried out by women with spontaneous menstruation. Considering as to the last four pregnancies, it is remarkable that to this group belonged a woman that had two successful childbirths; so the total number of women we followed was five (Fig. 1, Tab. 1). Average values of ferritin fluctuated between 500 and $1000 \mathrm{ng} / \mathrm{ml}$, and transaminases were between 25 and $100 \mathrm{U} / 1$. All of them were vaccinated against Hepatitis B Virus (HBV), and two were splenectomized, particularly the woman affected by primitive amenorrhoea and one of those with normal periods. Only the women affected by primitive amenorrhoea needed the induction of ovulation to stimulate her pregnancy. Evaluation of eligibility depends upon the results of a series of examinations regarding hearth, kidneys, thyroid, pancreas and peripheral arteries. Haemosiderotic parenchimal deterioration, particularly of the myocardium, makes these patients potentially fragile, while the induction of ovulation may be a hard stress test even for a healthy woman.

Tab. 1. Table expressing the number of married women, the pregnancies, the medium age and the amount of each group, according to a subdivision in three categories - Normal menstruation, Secondary Amenorrhoea and Primitive Amenorrhoea - in reference to the total amount of the $\mathbf{1 1 6}$ patients we studied.

\begin{tabular}{|c|c|c|c|}
\hline $\begin{array}{l}\text { Menstruation } \\
\text { characteristics }\end{array}$ & $\begin{array}{c}\text { Normal } \\
\text { menstruation }\end{array}$ & $\begin{array}{c}\text { Secondary } \\
\text { amenorrhoea }\end{array}$ & $\begin{array}{c}\text { Primary } \\
\text { amenorrhoea }\end{array}$ \\
\hline married women & 12 & 6 & 5 \\
\hline number of pregnancy & $4(30 \%)$ & $1(16.6 \%)$ & $1(20 \%)$ \\
\hline spontaneous pregnancy & $4(100 \%)$ & $1(100 \%)$ & - \\
\hline induced pregnancy & - & - & $1(100 \%)$ \\
\hline medium age & $23.6 \pm 2.4$ & $24.7 \pm 1.6$ & $26.1 \pm 3.2$ \\
\hline
\end{tabular}

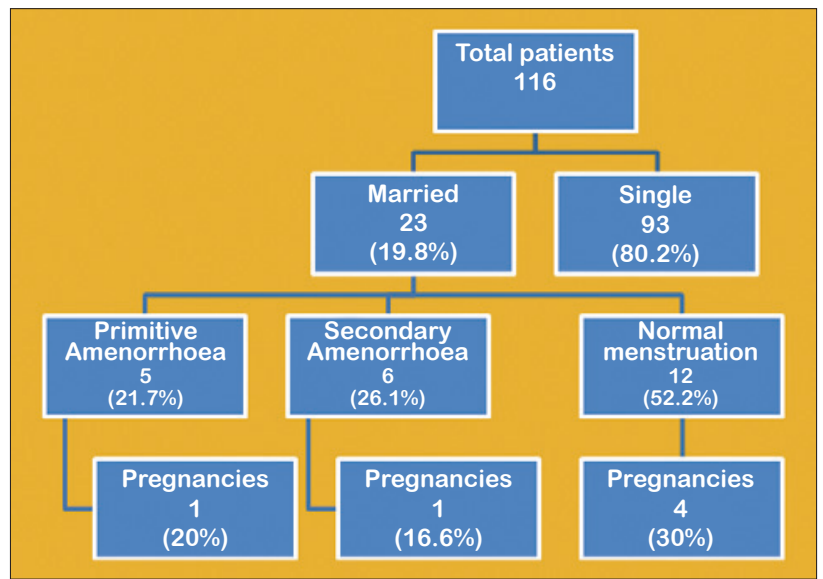

Fig. 1. Flow chart representing the distribution of 116 women affected by Beta Thalassemia major subdivided in three categories, according to their menstruation characteristics; considering if they are married or singles, and the number of pregnancies carried out by each group.

However by the advanced cardiac magnetic resonance imaging technique, early diagnosis of heart iron will allow the currently available iron-chelating agents (oral and parenteral) to be used in an innovative way to improve the quality of life and improve survival of patients with beta-thalassemia (10).

All the other women had spontaneous and physiological pregnancies. It is finally important to underline that all the partners of these women were negative as regards the thalassemic trait.

According to the guidelines previously established by Tuck (11), a precise and detailed pre-pregnancy assessment was effected before each conception. This was constituted by a series of clinical and laboratory essays, including checks for diabetes and hypothyroidism, for B and $\mathrm{C}$ hepatitis and for blood group antibodies. Cardiac function was assessed symptomatically and by echocardiography and exercise electrocardiography. Rubella immunity was checked and immunisation was given when the woman was found to be susceptible. During pregnancy the monitoring of all these factors continued, including frequent checks of aminotransferase (AST and ALT transaminases).

The frequency of blood transfusion had to be increased, the aim being to maintain the pre-transfusion haemoglobin concentration. Deferoxamine therapy was stopped as soon as pregnancy was diagnosed, because using this drug during gestation could notoriously induce teratogenic effects on the foetus, but the average values of ferritin were just a little higher than the normal range observed. Probably the foetus, due to a particular chelating activity, maintains it during gestation. This therapy was quickly resumed after delivery (12). Other pregnancy monitoring, such as for blood pressure, for proteinuria and for fetal growth, and cares during labour and delivery were effected according to usual obstetrics practice and criteria.

\section{Results}

Only one of the six pregnancies we have followed needed the induction of ovulation. Moreover a same woman carried out two of them. All the women were in labour when she were 38 week 
pregnant, and the outcome were six healthy babies born at term weighting between 2600 and $3300 \mathrm{gs}$. The caesarean delivery was preferred because of the frequent "infertility/sterility" that occurs in the natural history of such kind of patients. All of these women were neither HIV positive nor suffered from diabetes, however they were controlled with a diet. All of them reported transaminases raising, but only in one case it was necessary to practice a therapy based on silimarina. Moreover all of them had transfusion-transmitted $\mathrm{C}$ hepatitis prior to pregnancy, but only the one affected by secondary amenorrhoea received curses of interferon two years before embarking on pregnancy, because she showed to be positive for HCV-RNA. Five out of the babies born were HCV negative, while only one was HCV positive but HCV-RNA negative, so that it was not necessary to prescribe a therapy.

Finally, there were neither cardiac dysfunctions nor kidney diseases reported, or other important affections.

\section{Discussion}

We believe that several ethical and theoretical questions should be evaluated before the observation of technical problems. We should never forget that Beta thalassemic are fragile patients who are knowingly exposed to a large quantity of risks.

Several hemodynamic changes during pregnancy affect cardiac function and are known to cause an increase in maternal cardiac stress in the gestational period. An increase in blood volume, decrease in systemic vascular resistance, and augmentation of cardiac output occurs in all normal pregnancies to match maternalfetal demands (13). In one study of echocardiography in pregnant $\beta$-thalassemia patients without cardiac impairment, an appropriate increase in cardiac output was observed secondary to an increase in resting heart rate and left ventricular mass (14). Although cardiac function did appear to return to pre-pregnancy levels after delivery, a subset of $\beta$-TM patients was noted to have a late decline in cardiac reserve in the years after gestation; however, the long-term outcome for these patients is unknown.

More recent reports in $\beta$-TM patients without cardiac impairment and treated with aggressive pre-pregnancy chelation have generally observed favorable outcomes. One of the largest modern studies of 58 pregnancies in $\beta$-TM mothers found that impaired glucose tolerance and gestational diabetes were frequent complications, occurring in $15 \%$ of patients (15). Worsening of anemia during pregnancy also led to an increase in transfusion requirements of a median of $25 \mathrm{~mL} / \mathrm{kg} / \mathrm{y}$ and a subsequent $60 \%$ increase in baseline ferritin. Fetal complications such as IUGR and premature delivery did appear to be increased in $\beta$-TM patients, occurring in nearly $40 \%$ of infants; however, because many of these women required fertility treatments, twin pregnancies accounted for a major number of these births.

In $\beta$-TI, the most significant adverse maternal outcome appeared to be thrombotic events, which occurred in $7.2 \%$ of 83 pregnancies in one series (16). Development of anemia resulted in a need for transfusion in $60-80 \%$ of $\beta$-TI patients, $30 \%$ of whom had never had a transfusion before pregnancy $(15,16)$. IUGR and prematurity rates were also noted to be high among $\beta$-TI mothers, occurring in $20 \%$ and $30 \%$ of infants, respectively (16).
It is the doctor's responsibility to decide which patient can afford ovulation induction, when needed, pregnancy and labour, with an acceptable degree of risk, and which are the limits of this risk.

In conclusion with improved life expectancy in the hemoglobinopathies, many women with these disorders are now choosing to become pregnant. Although numerous complications can occur, vigilant monitoring by both experienced obstetricians and hematologists can lead to successful pregnancy outcomes.

Pregnancy does not have a deleterious effect on the course of their disease, and on the general wellness of the foetus. It seems, in fact, that the evaluation of cardiac function, in combination with a low iron load and satisfactory endocrine and hepatic controls can ensure a pregnancy outcome with minimal adverse effects, and not severe obstetric complications except for the high incidence of caesarean sections (17) and the risk of thrombotic events (16).

\section{References}

1. Galanello R, Origa R. Beta-thalassemia. Orphanet J Rare Dis. 2010; 5: 11. Published online 2010 May 21. doi: 10.1186/1750-1172-5-11.

2. Guide lines for the clinical management of thalassemia. 2nd ed. Nicosia Cyprus: Thalassemia International Federation; 2009. World Bank 2006, reported joint WHO - March of time meeting 2006-Quoted; p. 9.

3. Nadkarni A, Caraskar AC, Krishnamoorty R, Lu CY, Ghosh K, Colah R et al. Molecular pathogenesis and clinical variability of $\beta$ thalassemia syndrome among Indians. Am J Hematol 2001; 68: 75-80.

4. Karagiorga-Lagana M. Fertility in Thalassemia: The Greek Experience. J Pediatr Endocrinol Metab 1998; 11: 945-951.

5. Jensen CE, Tuck SM, Old J et al. Incidence of endocrine complications and clinical disease severity related to genotype analysis and iron overload in patients with beta-thalassaemia. Eur J Haematol 1997; 59 (2): 76-81.

6. De Sanctis V. Growth and puberty and its management in thalassemia. Horm Res 2002; 58: 72-79.

7. Kumar RM, Rizk DE, Khuranna A. Beta-Thalassemia major and successful pregnancy. J Reprod Med 1997; 42 (5): 294-298.

8. Tampakoudis P, Tsatalas C, Mamopoulos M, Tantanassis T, Christakis JI, Sinakos Z, Mantalenakis S. Transfusion-dependent homozygous beta-Thalassaemia major: successful pregnancy in five cases. Eur J Obstet Reprod Biol 1997; 74 (2): 127-131.

9. Daskalakis GJ, Papageorgiou IS, Antsaklis AJ, Michalas SK. Pregnancy and homozygous beta thalassaemia major. Br J Obstet Gynaecol 1998; 105 (9): 1028-1032.

10. Wonke B. Clinical management of beta-thalassemia major. Semin Hematol 2001; 38 (4): 350-359.

11. Tuck SM, Jensen CE, Wonke B, Yardumian A. Pregnancy management and outcomes in women with Thalassemia major. J Paediatr Endocrinol Metab 1998; 11 (3): 923-928.

12. Pafumi C, Zizza G, Caruso S et al. Pregnancy outcome of a transfusiondependent thalassemic woman. Ann Hematol 2000; 79 (10): 571-573.

13. Hill CC, Pickinpaugh J . Physiologic changes in pregnancy. Surg Clin North Am 2008; 88 (2): 391-401.

14. Aessopos A, Karabatsos F, Farmakis D et al. Pregnancy in patients with well-treated beta-thalassemia: Outcome for mothers and newborn infants. Am J Obstet Gynecol 1999; 180 (2 Pt 1): 360-365.

15. Origa R, Piga A, Quarta G et al. Pregnancy and beta-thalassemia: An Italian multicenter experience. Haematologica 2010; 95 (3): 376-381.

16. Nassar AH, Naja M, Cesaretti C, Eprassi B, Cappellini MD, Taher A. Pregnancy outcome in patients with beta-thalassemia intermedia at two tertiary care centers, in Beirut and Milan. Haematologica 2010; 93 (10): 1586-1587.

Received December 2, 2010. Accepted January 27, 2013. 